\title{
Possibly drug-induced palpable migratory arciform erythema*
}

\author{
Fernando Luiz Teixeira Dantas ${ }^{1}$ \\ Isis Suga Veronez ${ }^{1}$ \\ Juliana Ribeiro Leitão ${ }^{1}$
}

\author{
Neusa Yuriko Sakai Valente ${ }^{1}$ \\ Priscila Kakizaki ${ }^{1}$ \\ Rafael Cavanellas Fraga ${ }^{1}$
}

DOI: http:/ / dx.doi.org/10.1590/abd1806-4841.20153624

\begin{abstract}
A bstract: Palpable migratory arciform erythema is an entity of unknown etiology, with few published cases in the literature. The clinical and histopathological features of this disease are difficult to be distinguished from those of Jessner's lymphocytic infiltration of the skin, lupus erythematous tumidus and the deep erythema annulare centrifugum. We describe here the first two Brazilian cases of palpable migratory arciform erythema. The patients presented with infiltrated annular plaques and erythematous arcs without scales. These showed centrifugal growth before disappearing without scarring or residual lesions after a few days. They had a chronic course with repeated episodes for years. In addition, these cases provide evidence of a drug-induced etiology.
\end{abstract}

Keywords: Abnormalities, drug-induced; Lupus erythematosus, cutaneous; Pseudolymphoma

\section{INTRODUCTION}

Palpable migratory arciform erythema (PMAE) is part of the set of lymphocytic infiltrations of the skin. Histologically, it is characterized by a perivascular and periadnexal T lymphocytic infiltrate. Moreover, it is considered to classify within the spectrum of pseudolymphomas, like Jessner-Kanof 's lymphocytic infiltration of the skin-(JK-LI), with which it shares histopathological features. ${ }^{1.2}$

We report two cases of PMAE, discuss the clinical differences between PMAE, JK-LI, lupus erythematous tumidus (LET) and other dermatoses, and comment on the possibility of PMAE being drug-induced.

\section{CASESREPORT}

\section{Case 1:}

A 60-year-old female patient had a two-year history of erythematous plaque in the cervical region, with centrifugal growth and central clearing. The plaque spontaneously disappeared after seven to ten days, without scarring. The patient reported burning sensation at the site of the plaque. The lesions pro- gressed, alternating outbreaks and remissions at different sites, such as the chest and back. However, they always show the same pattern, at 20-to-30-day intervals. At the time of consultation, the patient presented with a lesion in the upper limb, which was $8 \mathrm{~cm}$ in diameter (Figure 1).

She was being treated for fibromyalgia and osteoarthritis with clomipramine, nimesulide and paracetamol.

Complete blood count, tests of liver and kidney functions all gave results within normal ranges. Antinuclear factor, as well as serology for herpes simplex virus, syphilis, and Borrelia burgdorferi were all negative. The patient underwent incisional biopsy of the arm lesion, which revealed predominantly lymphocytic perivascular and periadnexal dermatitis with eosinophils and neutrophils, and moderate mucin deposition in the dermis (Figure 2).

Drugs were discontinued. After a few weeks of remission, the lesions reappeared in acral regions (Figure 3). 


\section{Case 2:}

A 47-year-old female patient had a 1-year history of infiltrated, circinate erythematous lesions with elevated borders in the upper limbs, upper back and posterior neck. The lesions spontaneously disappeared after progressive central clearing and an average duration of three weeks. They are recurrent and migratory. (Figure 4) The patient made daily use of statin and consumed dipyrone and diclofenac sporadically. A biopsy of the right arm lesion with revealed lymphocytic perivascular and periadnexal dermatitis (Figure 5). In addition, Alcian Blue staining revealed a moderate amount of mucin deposition. PAS staining showed no thickening of the basement membrane (Figure 6).

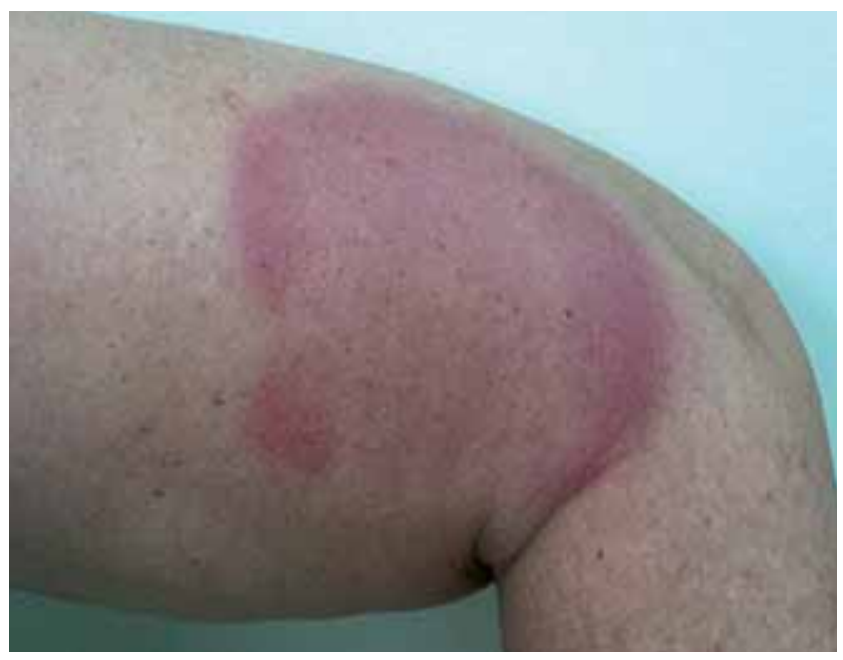

Figure 1: case 1 - lesion on the left arm. Arciform, elevated, infiltrated and erythematous plaque with mild erythema in the central area and $8 \mathrm{~cm}$ in diameter

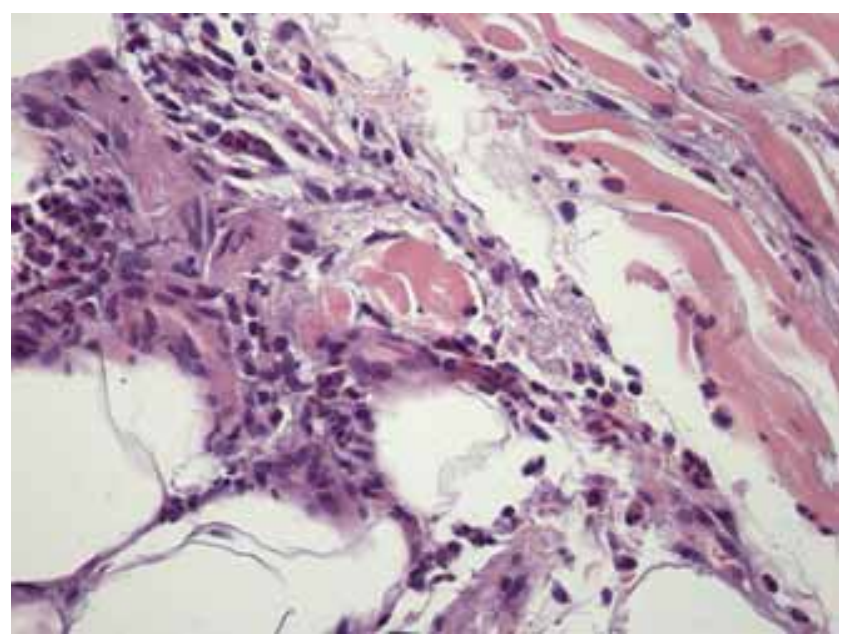

FIGURE 2: Histopathology of case 1 - hematoxylin-eosin, 400x magnification. Detail of a predominantly lymphocytic, mixed infiltrate with neutrophils and eosinophils in the periadnexal fat
After 12 weeks of drug discontinuation, there was complete regression of all lesions.

\section{DISASSION}

PMAE has very different evolution from that of JK-LI. The former leads to the emergence of dynamic skin lesions that increase in size and disappear after a few days. The appearance of new lesions in different areas justifies the use of the term "migratory". This
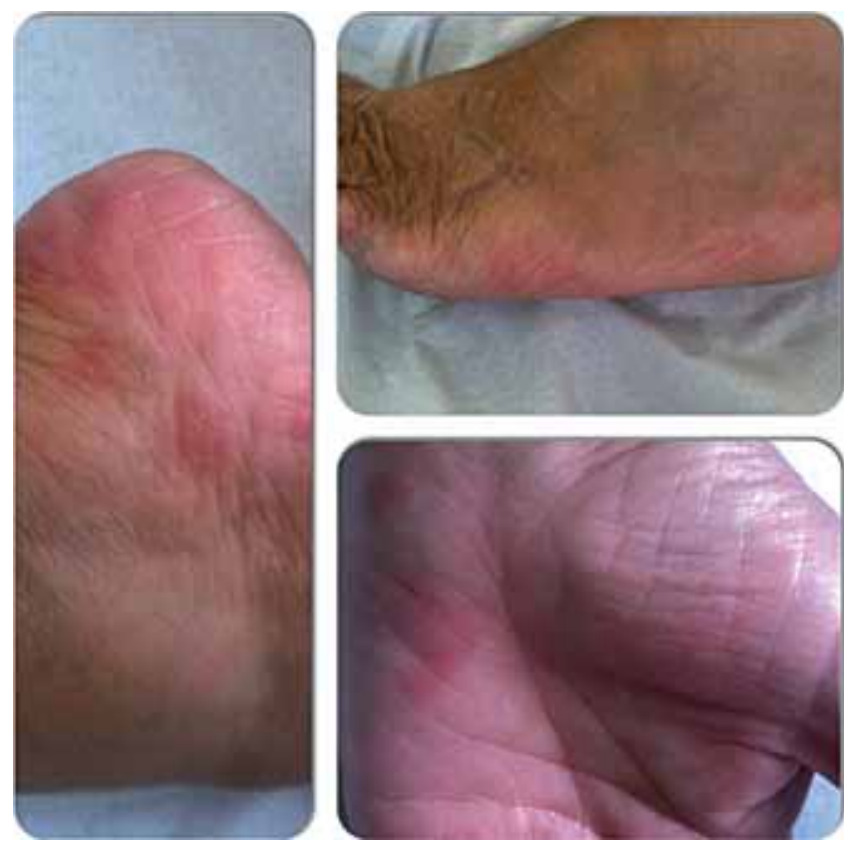

Figure 3: case 1 - acral lesions. Arciform, palpable, erythematous plaques in the hypothenar region of the hand, on the heel and lateral border of the foot

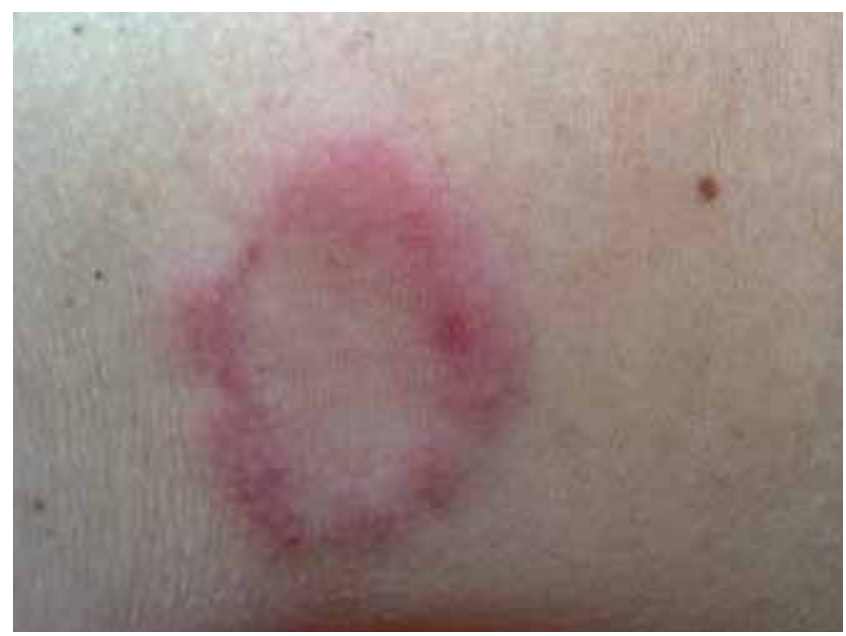

Figure 4: case 2 - lesion on the right arm. Annular, erythematous, infiltrated and palpable plaques with spared center on the lateral region of the arm 


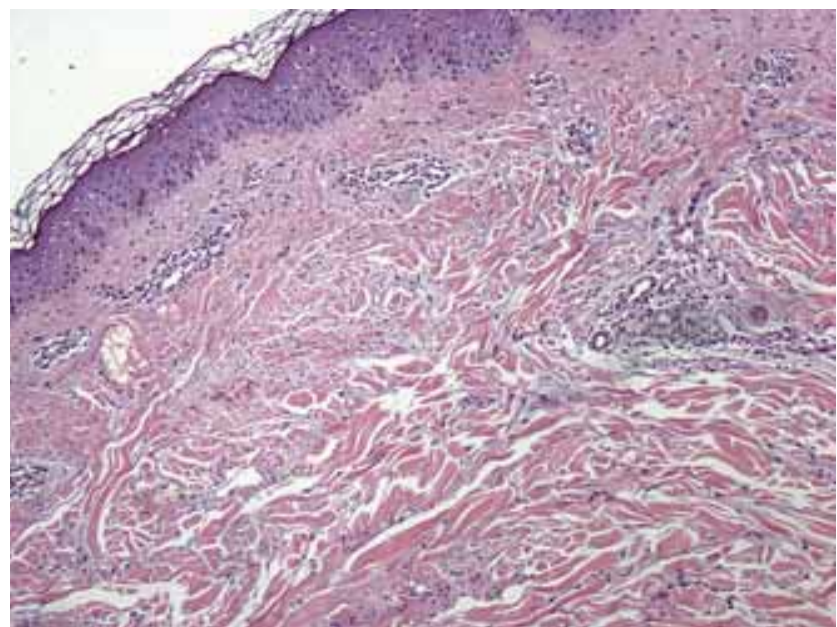

FIGURE 5: Histopathology of case 2 - hematoxylin-eosin, 100x magnification. Mildly acanthotic epidermis. Dermis with perivascular lymphocytic infiltrate and moderate interstitial mucinosis

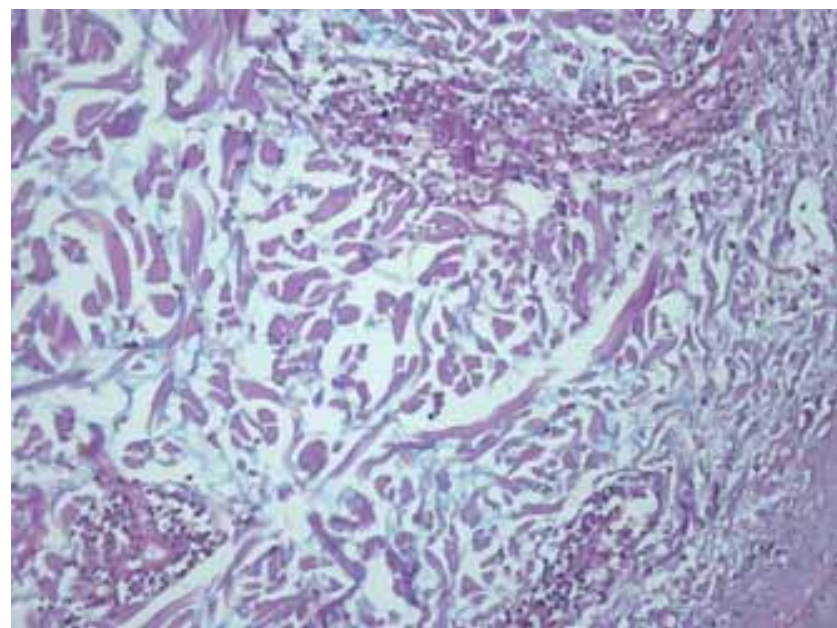

Figure 6: Histopathology of case 2 - OM x 200. Alcian blue staining counter-stained with PAS show moderate interstitial mucin deposition in the reticular dermis

feature is not shared by JK-LI, which presents static lesions that may persist for months to years without migrating to other areas of the body. Another differential feature of PMAE is the fact that the preferred areas of involvement are the back and arms, and less frequently the chest and thighs. There are no reports of cases on the face, the most common site affected by JK-LI. ${ }^{3.4}$

Histopathologic findings show a moderate to severe perivascular and periadnexal $\mathrm{T}$ lymphocytic infiltrate with no major epidermal alterations. Inflammatory cells have no interstitial distribution. Plasma cells are absent, which helps differentiate the disease from chronic erythema migrans (ECM). ${ }^{4}$

The pathogenesis is unknown and the polyclonality of the infiltrate speaks against a possible neoplastic nature. ${ }^{2,4}$ Furthermore, no evidence of infection by Treponema pallidum, Borrelia burgdorferi, and herpes simplex virus was found, which makes the infective hypothesis less likely. As there have been reports of drug-induced JK-LI (by leflunomide, subcutaneous injections of glatiramer acetate and inhibitors of angiotensin-converting enzyme), PMAE could eventually be a drug eruption, since the patients reported here were being treated with various drugs and there is a temporal relationship between the use of these drugs and the appearance of skin lesions. Additionally, we found a reasonable amount of eosinophils in the inflammatory infiltrate of the first patient and vacuolar degeneration of basal cells in the second patient, and the latter showed complete remission of PMAE after drugs discontinuation..$^{5-7}$ Current knowledge indicates that drugs may alter immune surveillance, leading to an abnormal activity of $\mathrm{T}$ lymphocytes, both in their differentiation and in their proliferation, which would cause cutaneous lymphocytic infiltrates or T lymphocytes pseudolymphomas. ${ }^{7}$

The differential diagnosis includes LET, the deep erythema annulare centrifugum (EAC), ECM, granuloma annulare and polymorphous light eruption. ${ }^{2,4,8}$ LET is clinically indistinguishable from JK-LI, since both entities share the same age of onset and histopathological findings, and show photosensitivity, absence of extra-cutaneous involvement, and good response to antimalarials. A mild epidermal atrophy appears to be present in the first and not the latter. Moreover, it is known that both may show mucin deposition in the dermis in $50 \%$ of cases, which does not help in differentiating between them and PMAE. ${ }^{9}$ Deep EAC also has the same clinical and pathological features as JK-LI, LET and PMAE. However, only the latter is characterized by migratory and fleeting lesions, while the others are conspicuous by the presence of lesions that last for months to years. ${ }^{10} \mathrm{ECM}$ was discarded due to negative serology for B. burgdorferi. Polymorphous light eruption also was also excluded because the lesions developed in non-photo-exposed areas. The hypothesis of granuloma annulare is incompatible with the histopathologic findings of the cases presented here.

Treatment of PMAE includes antimicrobials such as penicillin and cefuroxime, topical corticosteroids (either under occlusion or not) and UVA-1 phototherapy, in an attempt to prevent outbreaks or extend the time between them. Unfortunately, most cases tend to recur after weeks, despite treatment attempts. ${ }^{2,4,8}$ 
In this paper, we describe the first two Brazilian cases of PMAE whose etiology involves several hypotheses and to which we add the induction by drugs. In addition, as the number of cases reported in the literature is small, few conclusions can be made. Further studies might show whether PMAE, JK-LI, LET and the deep EAC are part of a continuous spectrum of clinical variants of the same entity.

\section{REFERENCES}

1. Jessner M, Kanof NB. Lymphocitic infiltration of the skin. AMA Arch Derm Syphilol. 1953;68:447-9.

2. Lohrisch I, Alexandrakis E, Maywurm H, Petres J. Erythema migrans arciforme et palpable (T-Zell Pseudolymphom). Hautarzt. 1990;41:78-82.

3. Steinmann A, Gummer M, Agathos M, Breit R. Palpable migratory arciform erythema and lymphocitic infiltration of the skin - different presentations of the same entity? Hautarzt. 1999;50:270-4.

4. Abeck D, Ollert MW, Eckert F, Szeimies RM, Tiemann M, Braun-Falco 0, et al. Palpable migratory arciform erythema. Clinical Morphology, histopathology, immunohistochemistry, and response to treatment. Arch Dermatol. 1997;133:763-6.

5. Sparsa L, Afif N, Goetz J, Sordet C, Chatelus E, Lipsker D, et al. Jessner-Kanof induced by leflunomide: a dermal variant of cutaneous lupus? Rheumatol Int. 2011;31:255-8.

6. Nolden S, Casper C, Kuhn A, Petereit HF. Jessner-Kanof lymphocytic infiltration of the skin associated with glatiramer acetate. Mult Scler. 2005;11:245-8.

7. Schepis C, Lentini M, Siragusa M, Batolo D. ACE-inhibitor-induced drug eruption resembling lymphocytic infiltration of the skin (of Jessner-Kanof) and lupus erythematosus tumidus. Dermatology. 2004;208:354-5.

8. Wagner G, Bartsch S, Rose C, Sachse MM. Palpable migratory arciform erythema. Hautarzt. 2012;63:965-8.

9. Rémy-Leroux V, Léonard F, Lambert D, Wechsler J, Cribier B, Thomas P, et al. Comparison of histopathologic-clinical characteristics of Jessner's lymphocytic infiltration of the skin and lupus erythematosus tumidus: multicenter study of 46 cases. J Am Acad Dermatol. 2008;58:217-23.

10. Nayak SU, Shenoi SD, Prabhu SM. Erythema annulare centrifugum: deep variant with vasculitis like histopathology. Indian J Dermatol. 2013;58:329.

\author{
M AILING ADDRESS: \\ Fernando Luiz Teixeira D antas \\ Rua Borges Lagoa, 1755 - Vila Clementino \\ 04038-034 - São Paulo - SP \\ Brazil \\ E-mail: fernandotdantas@uol.com.br
}

How to cite this article: Dantas FLT, Valente NY, Veronez IS, Kakizaki P, Leitão JR, Fraga RC. Possibly druginduced palpable migratory arciform erythema. An Bras Dermatol. 2015;90 (3 Suppl 1): S77-80. 